\title{
THE EMERGENCE OF NETWORK COMMUNITIES BY THE ACTION OF COEVOLVING MARKET AGENTS
}

\author{
D. Horváth, Z. Kuscsik \\ Department of Theoretical Physics and Astrophysics, Šafárik University, Košice, Slovak Republic
}

\begin{abstract}
The agent-based multileader model of the stock price dynamics on the directed evolving complex network is studied by direct simulation. The resulting stationary regime follows from the balance of extremal dynamics, adaptivity of the strategic variables and reconnection rules. For the given parametric combination the network displays a small-world phenomenon with high clustering coefficients and power-law node degree distribution. The fitness exploration by the mechanism of repeated random walk is used to violate dominance of centralized leadership. The simulation suggests that ultraslow dynamics of fitness implies explanation of the long-time volatility of the log-price returns.
\end{abstract}

Агентно-ориентированная мультилидерная модель динамики цен фондового рынка на основе направленной эволюционирующей сложной сети исследуется средствами прямого моделирования Получающийся в результате стационарный режим определяется балансом экстремальной динамики, адаптивности оперативных переменных и правилами перестройки соединений. Для заданной параметрической схемы сеть проявляет свойства так называемого явления малого мира с большими коэффициентами кластеризации и степенным законом распределения узлов. Для устранения преобладания централизованного лидера соответствие исследуется с помощью повторяющегося процесса случайного блуждания. Моделирование предполагает, что ультрамедленная динамика соответствия заключает в себе объяснение долговременной волативности логарифмических приращений цены.

PACS: 89.65.Gh; 89.75.Hc; 05.65.+b

\section{INTRODUCTION}

The design of decentralized systems of autonomous interacting agents with abilities to automatically devise societies so as to accommodate their behaviour via social and economic norms in emergent ways is highly challenging. As a general framework for starting of statistical considerations of interacting agents that act in a way to increase their own utility we take the Ising model. Looked at from the perspective of the macroeconomics, the model has a great importance, because it demonstrates that a basic interaction between the spins (also agents) can bring nontrivial collective phenomena. The parallels between fluctuations in the economic and magnetic systems afford an application of Ising model to the stock market statistics [1]. Particularly important for socioeconomic applications are works [2,3] setting the context with the minority game theory [4]. It has been confirmed that the spin analogs are able to shed light on aspects of financial data complexity. In our present formulation we incline closer to the spin variable reinterpretation $[3,5]$. We concretize the concept by defining the system of $L$ interacting traders (spins, agents) that occupy enumerated sites 
(nodes) $i=1,2, \ldots, L$ on lattice (or network) $\Gamma$. The agent's state is defined by spin variable $S^{(t)}(i) \in\{-1,0,1\}$; the upper index $(t)$ of $S^{(t)}(i)$ labels the market time. Each agent has an attitude to place buy order $S^{(t)}(i)=1$, sell order $S^{(t)}(i)=-1$ or stay inactive $\left[S^{(t)}(i)=0\right]$. The variable $S^{(t)}(i)$ is updated by stochastic dynamics according to local effective field. Naturally, the reinterpretation of magnetization $m^{(t)}=\frac{1}{L} \sum_{i=1}^{L} S^{(t)}(i)$ has been introduced.

According to it, the predominance of buy orders manifests itself throughout $m^{(t)}>0$, while $m^{(t)}<0$ corresponds to under-valued stock. The view assumes direct proportionality between the logarithm of the stock price returns $\ln \left[p^{(t+1)} / p^{(t)}\right]$ and $m^{(t+1)}-m^{(t)}$.

\section{THE CO-EVOLUTIONARY MODEL}

An attempt we use here is roughly analogous structurally dynamic cellular automata [6] in which the conventional rules are generalized to formalism where geometry and matter are dynamically coupled. We assume that the behaviour of market is determined by the underlying complex network defined by dynamical rules for links between buyers and sellers. Formally, the stochastic co-evolutionary dynamics of agents is described by the recursive formula

$$
\bar{\Pi}^{(t+1)}=\hat{\mathbf{U}}\left(\bar{\Pi}^{(t)}\right)
$$

including the composed configuration $\bar{\Pi}^{(t)} \equiv\left\{\Pi^{(t)}(1), \Pi^{(t)}(2), \ldots, \Pi^{(t)}(L)\right\}$ that consists of intra-agent spins $\Pi_{s s}^{(t)}(i) \equiv\left\{s^{(t)}(i, q)\right\}_{q=1, \ldots, N_{\text {intr }}}$, with $s^{(t)}(i, k) \in\{-1,1\}$; strategic variables $\Pi_{J}^{(t)}(i)=\left\{J_{\text {intr }}^{(t)}(i, k, q), J_{\text {ext }}^{(t)}(i), h_{0}^{(t)}(i), \kappa^{(t)}(i)\right\}$, where $k, q \in\left\{1,2, \ldots, N_{\text {intr }}\right\}$, network links $\Pi_{X}^{(t)}(i) \equiv\left\{X_{n}^{(t)}(i)\right\}, n \in I_{\text {out }} \equiv\left\{1,2, \ldots, N_{\text {out }}\right\}$. The nonlinear operator $\widehat{\mathbf{U}}$ acts as an arrangement of single-agent operators of: (a) local field $\widehat{U}_{\mathbf{s s}}(i)$ acting on $\Pi_{s s}(i)$; (b) adaptivity $\widehat{U}_{\mathbf{A d}}\left(i_{a}\right)$ acting on $\Pi_{J}\left(i_{a}\right), i_{a} \in \Gamma$; (c) reconnection $\widehat{U}_{\mathbf{R e}}\left(i_{r}\right), i_{r} \in \Gamma$, acting on $\Pi_{X}^{(t)}\left(i_{r}\right)$; (d) extremal dynamics $\widehat{U}_{\mathbf{E x}}\left(i_{\min F}\right)$ acting on $\Pi_{s s}\left(i_{\min F}\right)$ and $\Pi_{J}\left(i_{\min F}\right)$, where $i_{\min F}=\arg \min _{j \in \Gamma} F(j)$ belongs to agent with a minimum of the fitness $F(\cdot)$ (see definition Eq. (3)). The comments to the role of above variables are provided in below.

Suppose the directed network of labeled nodes $\Gamma \equiv\{1,2, \ldots, L\}$, where node $i \in \Gamma$ attaches via $N_{\text {out }}$ directed links to its neighbors $X_{n}(i) \in \Gamma, n \in I_{\text {out }}$, i.e., the graph is $N_{\text {out }}$-regular. Two outgoing links $X_{1}(i)=1+i \bmod L, X_{2}(i)=1+(L+i-2) \bmod L$ of node $i$ create the bidirectional cycle static subgraph ( $L$-gon). This guarantees the preservation of connectedness at any stage $t$. The reconnection rules are applied exceptionally to the links $X_{n}^{(t)}(i), 3 \leqslant n \leqslant N_{\text {out }}$. The links are mediators of the $J_{\text {ext }}^{(t)}(i)$ pair couplings through which agent $i$ reacts to game-relevant information about the sell/buy orders of linked neighbors. The random field term $h_{0}^{(t)}(i)$ expresses the effort to preserve decision sign $\left(h_{0}\right)$, the term $\kappa^{(t)}(i) m^{(t)}$ synchronizes actions of agents. The novel feature of the model presented in this paper is that it goes beyond the elementary single-spin-single-agent conception. The intraagent small-scale fully connected intranet is considered with the variables $J_{\text {intr }}^{(t)}(i, \ldots)$ slowly tuned by the self-organizing learning governed by adaptivity principles. Thus, the whole complexity of agent is bounded by $2^{N_{\text {intr }}}$ states allowed by $\Pi_{s s}(i)$. 
The effective local field $h_{s s}^{(t)}(i, k)$ is defined as a superposition of the large-scale (interagent) and small-scale (intra-agent intranet) contributions

$$
\begin{aligned}
\widehat{U}_{\text {ss }}(i): \quad h_{\text {ss }}^{(t)}(i, k) \leftarrow \frac{J_{\text {ext }}^{(t)}(i)}{N_{\text {out }}} & \sum_{n \in I_{\text {out }}} S^{(t)}\left(X_{n}^{(t)}(i)\right)+\kappa^{(t)}(i) m^{(t)}+h_{0}^{(t)}(i)+ \\
& +h_{\text {stoch }}^{(t)}(i, k)+\frac{1}{N_{\text {intr }}-1} \sum_{q=1, q \neq k}^{N_{\text {intr }}} J_{\text {intr }}^{(t)}(i, k, q) s^{(t)}(i, q),
\end{aligned}
$$

where $h_{\mathrm{stoch}}^{(t)}(i, k)$ is the Gaussian stochastic variable of dispersion $\sigma_{\mathrm{h}_{\text {stoch }}}$ and $J_{\text {intr }}^{(t)}(i, k, q)$ is the system of $N_{\text {intr }} \times N_{\text {intr }}$ pair couplings. The time step belongs to asynchronous spin update $s^{(t+1)}(i, k) \leftarrow \operatorname{sign}\left(h_{s s}^{(t)}(i, k)\right)$. Two-neuron output of intranet is considered with neurons $s^{(t)}(i, 1), s^{(t)}(i, 2)$. We designed the intranet architecture where the agent's sell/buy order $S^{(t)}(i) \leftarrow \frac{1}{2}\left[s^{(t)}(i, 1)+s^{(t)}(i, 2)\right]$, where $1 / 2$ yields state space $\{-1,0,1\}$ of $S^{(t)}(i)$.

The principles governing the co-evolution enter through the local fitness (score, popularity) that expresses an ability/inability to survive in the competitive environment. The local fitness is defined in an indirect manner as an integral over the history of orders

$$
F^{(t+1)}(i) \leftarrow F^{(t)}(i)-S^{(t)}(i) m^{(t)}
$$

The dynamics of $\Pi_{J}^{(t)}$ comes from the concept that real agents are partially rational [7]. The main principle we pursue is the mechanism of adaption. According to it, the agent $i_{a}$ starts with the choice of the prototype node $i_{\text {prot }}=X_{n_{a}}\left(i_{a}\right)$ (with randomly picked $n_{a} \in I_{\text {out }}$ ). The adaption to prototype is described by updates

$$
\widehat{U}_{\mathbf{A d}}\left(i_{a}\right): \quad \mathcal{J}^{(t+1)}\left(i_{a}\right) \leftarrow \mathcal{J}^{(t)}\left(i_{a}\right)(1-\eta)+\eta \mathcal{J}^{(t)}\left(i_{\text {prot }}\right)
$$

performed for alternatives $\mathcal{J}^{(t)}(i)$ from the tuple $\Pi_{J}^{(t)}(i) ; \eta \in(0,1)$ is the plasticity parameter.

Recently, the research in complex networks has been extended to the local rules governing dynamics of social and technological networks [8]. The core of the most approaches is grounded on mechanism of preferential attachment [9]. The reason for the revision of our antecedent concept of attachment to single leader [10] is that the larger the market is, the more demanding the effort of agent to localize an unique leader has to be. Here, we are testing the indirect method for generation of net with varying number of leaders. The process is modeled by random walk on the net [11]. The information involved in $\left\{F(i) ; i \in \operatorname{RRW}\left(i_{r}\right) ; i \in \Gamma\right\}$ is gathered by random walker performing the repeated random walk (RRW) supplemented by the search for agent $\left(i_{B}\right)$ that holds highest locally recognizable fitness. The $\operatorname{RRW}\left(i_{r}\right) \subset \Gamma$ is constructed by including $N_{\text {path }}$ steps $N_{\text {rep }}$ times repeated from the actual origin $i_{r} \in \Gamma$. The reconnection consists of edge $X_{n_{W}}^{(t)}\left(i_{r}\right), n_{W} \equiv \arg \min _{n \in I_{\text {out }}} F\left(X_{n}^{(t)}\left(i_{r}\right)\right)$ disconnection composed with subsequent connection to the locally «best» node $i_{B}\left(i_{r}\right) \in \Gamma$

$$
\widehat{U}_{\mathbf{R e}}\left(i_{r}\right): \quad X_{n_{W}}\left(i_{r}\right) \leftarrow i_{B} \equiv \arg \max _{j \in \operatorname{RRW}\left(i_{r}\right)} F(j), \quad \operatorname{RRW}\left(i_{r}\right) \equiv \bigcup_{z=1}^{N_{\text {rep }}} \operatorname{path}_{z}\left(i_{r}\right),
$$


where $\operatorname{path}_{z}\left(i_{r}\right) \equiv\left\{\bar{i}_{1, z}\left(i_{r}\right), \ldots, \bar{i}_{N_{\text {path }}, z}\left(i_{r}\right)\right\} \subset \Gamma$ is composed of iterations $\bar{i}_{p}=X_{n_{p}}^{(t)}\left(\bar{i}_{p-1}\right)$, $\bar{i}_{0} \equiv i_{r}$ with random $n_{p} \in I_{\text {out }},\left(p=1,2, \ldots N_{\text {path }}\right)$. Moreover, $\widehat{U}_{\mathbf{R e}}\left(i_{r}\right)$ is conditioned by the requirement that no multiple links can attach $i_{B}$ from $i_{r}$, while $X_{n}\left(i_{r}\right) \neq i_{r}$ for all $n \in I_{\text {out }}$.

Further mechanism decisive for co-evolution is extremal dynamics [12]. Here extremality means that the instant value of the strategic variable owned by node $i_{\min F}$ is immediately replaced by Gaussian distributed random numbers $\mathrm{N}\left(0, \sigma_{\ldots}\right)$ of dispersion $\sigma_{\ldots}$ : $h_{0}\left(i_{\min F}\right) \leftarrow \mathrm{N}\left(0, \sigma_{h_{0}}\right), \quad \kappa\left(i_{\min F}\right) \leftarrow \mathrm{N}\left(0, \sigma_{\kappa}\right), \quad J_{\text {ext }}\left(i_{\min F}\right) \leftarrow \mathrm{N}\left(0, \sigma_{J_{\text {ext }}}\right)$, $J_{\text {intr }}\left(i_{\min F}, k, q\right) \leftarrow \mathrm{N}\left(0, \sigma_{J_{\text {intr }}}\right), F\left(i_{\min F}\right) \leftarrow \mathrm{N}\left(0, \sigma_{\mathrm{F}}\right)$, generated according to dispersions $\sigma_{\kappa}, \sigma_{h_{0}}, \sigma_{J_{\text {ext }}}, \sigma_{J_{\text {intr }}}, \sigma_{\mathrm{F}}$

\section{SIMULATION RESULTS}

The selection of relevant combination within the parametric space is nontrivial task that would be the theme of our future work. To attain at least qualitative agreement with known economic patterns [13], the appropriate values $L=500, N_{\text {out }}=10, N_{\text {depth }}=6, N_{\text {depth }}=6$, $\sigma_{J_{\text {ext }}}=6, \sigma_{J_{\text {intr }}}=1, \sigma_{h_{0}}=4, \sigma_{\kappa}=1, \sigma_{h_{\text {stoch }}}=0.05, \sigma_{F}=0.1, P_{\text {Re }}=0.01, P_{\text {Ad }}=0.2$, $\eta=0.025$ have been inferred from parameter sweeps and extensive simulations. (Here $P_{\text {Re }}$, $P_{\text {Ad }}$ denote the probability of $\widehat{U}_{\mathbf{R e}}$ and $\widehat{U}_{\text {Ad }}$, respectively.) The particular requirement has been to keep the spin dynamics much faster than adaptivity that should be more frequent than reconnections. The additional requirement aims at attaining the vicinity of a critical point, where the power-law probability density functions (pdf's) are generated [14]. The statistics for calculation of averages has been collected from $3 \cdot 10^{7}$ Monte Carlo steps per node.

The node degree $k^{(\mathrm{in})}(j)=\sum_{i \in \Gamma} \sum_{n \in I_{\mathrm{out}}} \delta_{j, X_{n}(i)}$ accounts for incoming links of node $j$. The stationary regime permits the sequence of networks with broad-scale $\operatorname{pdf}\left(k^{(\text {in })}\right)$ described local exponents $\gamma^{\text {in }} \simeq-1.23$ (as $k^{\text {(in) }}>20$ ) of the dependence $\operatorname{pdf}\left(k^{\text {in }}\right) \sim\left[k^{(\text {in })}\right]^{\gamma^{\text {in }}}$. The valuable information on network structure is provided by the clustering coefficient $C(i)$ [16], which expresses the strength of social relationships - social transitivity [17]. Customarily, it is meaningful to compare the mean value $\langle C\rangle$ of distinct network reconnection modes. For partially random net (only the links $n \geqslant 3$ are randomized) we obtained $\left\langle C_{\text {rand }}\right\rangle \simeq 0.02$, while $\langle C\rangle \simeq 0.51$, and thus $\langle C\rangle /\left\langle C_{\text {rand }}\right\rangle \simeq 25.5$. For comparative reasons, the average of minimum
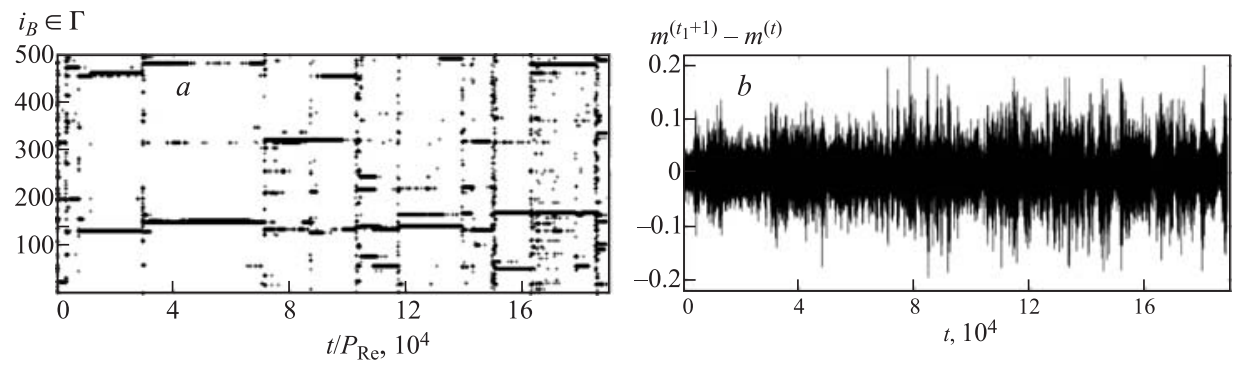

a) The time-dependence of $i_{B}$ (see Eq. (5)) shows the punctuated equilibrium. One can see how the epochs dominated by a group of leaders switch rapidly to epochs governed by few leaders. $b$ ) The corresponding time-dependence of the log-price returns 
path lengths have been computed for random net $\left\langle l_{\text {min,rand }}\right\rangle \simeq 2.9$. In contrast, $\widehat{U}_{\text {Re }}$ yields $\left\langle l_{\min }\right\rangle \simeq 16$. The combination of the above-mentioned network attributes indicates what is known as small-world behaviour. The main outcome of our analysis is plotted in the Figure. It confirms the emergence of time-varying multileader market with distributed leadership.

The statistical treatment of simulation data (see the Figure) leads to the fat-tailed pdf that can be fitted by the stretched exponential $\operatorname{pdf}(x) \simeq \exp \left(3.48-53.94|x|^{z}\right), z \simeq 1$ of the log-price returns [15]. The finding is in accordance with pdf's obtained for returns of financial indexes NASDAQ, DJIA and S\&P500. The time scales of simulated model can be highlighted by the autocorrelation functions of $m^{(t+1)}-m^{(t)},\left|m^{(t+1)}-m^{(t)}\right|, J_{\text {ext }}^{(t)}(i)$ and $F^{(t)}(i)$. The autocorrelations of $\operatorname{sign}\left(m^{(t)}\right)$ are significantly stronger than $m^{(t+1)}-m^{(t)}$ in conformity with empirics [18]. In addition, the analysis of $\left|m^{(t+1)}-m^{(t)}\right|$ confirms the volatility clustering [13]. Surprisingly, very slow time scale $\left(\sim 10^{4} \mathrm{MC}\right.$ steps $)$ belongs to $F^{(t)}(i)$.

\section{CONCLUSIONS}

The simultaneous production of the power-laws of the topological and price distributions has been attained as an exceptional consequence of extremal dynamics. Our general remark in this context is that indexes are parametrically dependent and it is disputable if universal in the context of phase transitions. The simulations confirmed that community (modular) building blocks can emerge due to combined effect of RRW and reconnections. There is a clear need for more comprehensive research that would systematize abundant macroscopic phases.

Acknowledgements. The authors would like to express their thanks to Slovak Grant agency VEGA (grant No.1/2009/05), grants IT2005/IT2006 and agency APVT-51-052702 for financial support.

\section{REFERENCES}

1. Cont R., Bouchaud J. P. // Macroecon. Dynam. 2000. V.4. P. 170;

Chowdhury D., Stauffer D. // Eur. Phys. J. B. 1999. V. 8. P. 477;

da Silva L.R., Stauffer D. // Physica A. 2001. V.294. P. 235.

2. Ponzi A., Aizawa Y. // Physica A. 2000. V.287. P. 507;

Bornholdt S. // Intern. J. Mod. Phys. C. 2001. V. 12. P. 667;

Kaizoji T., Bornholdt S., Fujiwara Y. // Physica A. 2002. V.316. P. 441.

3. Takaishi T. // Intern. J. Mod. Phys. C. 2005. V. 16. P. 1311.

4. Challet D., Marsili M. // Phys. Rev. E. 1999. V.60. P. R6271.

5. Greco A., Sorriso-Valvo L., Carbone V. cond-math0601047. 2006.

6. Ilachinski A., Halpern P. // Complex Syst. 1987. V.1. P. 503.

7. Gigerenzer G., Selten R. Bounded Rationality. Cambridge MIT Press, 2002.

8. Zhu H., Wang X., Zhu J. Y. // Phys. Rev. E. 2003. V. 68. P. 056121. 
9. Albert R., Barabási A.-L. // Rev. Mod. Phys. 2002. V.74. P. 47.

10. Horváth D., Kuscsik Z., Gmitra M. // Physica A. 2006. V.369. P. 780.

11. Bray A. J., Rodgers G. J. // Phys. Rev. B. 1988. V.38. P. 11461; Almaas E., Kulkarni R. V., Stroud D. // Phys. Rev. E. 2003. V.68. P. 056105.

12. Bak P., Sneppen K. // Phys. Rev. Lett. 1993. V.71. P. 4083.

13. Mantegna R.N., Stanley E.H. An Intoduction to Econophysics: Correlations and Complexity in Finance. Cambridge: Cambridge Univ. Press, 1999.

14. Solé R. V., Manrubia S. C. // Phys. Rev. E. 1996. V. 54. P. R42-R45.

15. Sornette D. Why Stock Markets Crash: Critical Events in Complex Financial Systems. Princeton Univ. Press, 2003; cond-mat/0301543. 2003.

16. Dorogovtsev S. N., Mendez J. F. F. cond-mat/0404593.

17. Ebel H., Davidsen J., Bornholdt S. // Complexity. 2003. V. 8. P. 24.

18. Lillo F., Mike S., Farmer J. D. // Phys. Rev. E. 2005. V.7106. P. 287. 